\title{
Déclinaison nominale en dan-gwèètaa (groupe mandé-sud, Côte-d'Ivoire) ${ }^{1}$
}

\author{
Valentin Vydrin*
}

\section{INTRODUCTION}

Le Dan-gwèètaa appartient au continuum dialectal dan dont les variantes sont parlées au Liberia (où elles sont connues sous le nom « Gio ») et en Côte d'Ivoire (où ils sont appelés «Yacouba »). Le dan-gwè̀taa a cinq tonèmes unis et trois tonèmes modulés dont les désignations sont (sur l'exemple de la voyelle $a$ ) : á extra-haut, á - haut, $\bar{a}$ - moyen, $\grave{a}$ - bas, $\ddot{a}$ - extra-bas, $\ddot{a}$ - extra-haut-descendant, $\hat{a}$-haut-descendant, $\vec{a}$ - moyen-descendant. À la différence de la plupart des autres langues mandé, les changements contextuels des tons en dan-gwèètaa sont minimaux. On compte douze voyelles orales (antérieures, postérieures nonarrondies, postérieures arrondies, quatre niveaux d'aperture pour chaque série) et neuf nasales. Les consonnes nasales n'ont pas de statut phonologique, elles représentent des allophones des sonantes et des implosives devant les voyelles nasales.

Le dan se caractérise par un ordre des mots fixe. Ainsi, dans une phrase transitive verbale, on a: (Sujet) - Marque Prédicative Pronominale ${ }^{2}$ Complément d'Objet Direct - Verbe - Complément d'Objet Indirect ou Oblique (- Postposition). La présence du sujet n'est pas obligatoire. Dans une phrase intransitive, le complément d'objet direct est absent. L'ordre des mots dans un

\footnotetext{
*LLACAN (Inalco, CNRS - UMR8135). vydrine@gmail.com

1 Mes remerciements vont au Prof. Thomas Bearth, sans qui ce projet ne serait pas possible, aux membres de la SIL-Côte d'Ivoire (surtout à Margrit Bolli) dont l'assistance nous a beaucoup facilité le travail de recherche en Côte d'Ivoire. Enfin j'ai une dette particulière à l'égard de feu M. Kessé Mongnan, mon informateur principal, dont la patience et la qualification linguistique ont beaucoup contribué à la réussite de notre projet, et de Diomandé Vassiafa (Bakari) avec qui j'ai travaillé après la mort de Kessé Mongnan.

2 Le terme «marque prédicative» enraciné dans la tradition linguistique mandé correspond au terme «auxiliaire» de la linguistique générale. Il faut cependant tenir compte du fait que les "marques prédicatives" ne sont pas du tout assimilables aux verbes dont elles se distinguent très nettement par leur comportement morphologique et syntaxique.
} 
groupe nominal de type génitif est « déterminant - déterminé », et dans un groupe attributif, « déterminé - déterminant ».

Par rapport aux langues les plus connues de la famille linguistique mandé, les langues du groupe mandé-sud sont généralement beaucoup plus «morphologiques », c'est tout particulièrement le cas pour le dan, notamment pour le nom.

Dans ce qui suit, il s'agira du gwèètaa, un des parlers nord-est du dan choisi comme base d'une des normes littéraires de cette langue. En analysant les données linguistiques dan-gwèètaa, on y trouve suffisamment de raisons de dire qu'un système de déclinaison du nom est en formation. Cela est assez extraordinaire: dans les langues niger-congo ${ }^{3}$, la déclinaison des noms est extrêmement rare, surtout en Afrique de l'Ouest où elle est pratiquement inexistante (König, 2008). Les données du dan-gwèètaa sont particulièrement intéressantes pour illustrer l'émergence de cas morphologiques à partir d'un système dénué de flexion.

Ce processus n'a pas touché l'ensemble des noms dan-gwè̀taa. En fait, il a eu pour résultat la division des noms en deux parties du discours différentes : les substantifs, qui n'ont pas la catégorie du cas grammatical, et une autre classe de lexèmes qui ont cette catégorie et auxquels je vais me référer ici comme « les noms locatifs $»^{4}$.

En 1., je commencerai ici par analyser la position des noms locatifs dans le système des parties du discours du dan-gwètaa. Comme l'existence même de déclinaison nominale dans une langue mandé est un fait exceptionnel, il semble nécessaire de présenter une description assez détaillée de la morphologie des « noms locatifs » pour fournir au lecteur suffisamment d'éléments sur la nature de la flexion nominale en dan-gwèètaa; cette présentation sera faite en 2. En 3., je fournirai quelques informations d'ordre syntaxique : l'accord en cas entre le nom locatif et la marque possessive; l'annulation de la déclinaison en présence d'attributs adjectivaux et de déterminatifs ; l'emploi des formes de cas obliques à l'intérieur du groupe nominal; le redoublement des noms locatifs. En 4., il s'agira d'une réflexion sur le sémantisme des noms locatifs (autrement dit, les conditions sémantiques favorisant l'entraînement des noms dans le système de déclinaison). En 5., j'analyserai quelques phénomènes d'autres langues mandé pouvant servir d'arrière-plan pour comprendre l'émergence de la déclinaison en dan. En conclusion, j'analyserai la déclinaison nominale en dan-gwèètaa du point de vue de la typologie du cas. Une liste de noms locatifs et de leurs formes obliques est présentée en annexe.

${ }^{3}$ Selon la classification de J. Greenberg, la famille mandé fait partie de la macrofamille niger-congo. Cette attribution n'étant pas confirmée par des reconstructions et correspondances régulières, elle a été mise en doute dans quelques publications récentes (en particulier, cf. Dimmendaal 2008). Comme ce problème n'est pas central pour le sujet de cet article, je n'en traiterai pas en détail.

4 Alternativement, on pourrait parler d'une seule partie de discours des «noms» subdivisées en deux classes morphologiques selon leur aptitude à la déclinaison. 


\section{NOMS LOCATIFS ET LES PARTIES DE DISCOURS CONTIGUËS}

Les noms locatifs sont, en dan-gwèètaa, beaucoup moins nombreux que les substantifs : ceux-ci se comptent par milliers, tandis que ceux-là se calculent par dizaines ${ }^{5}$.

Au plan morphologique, les noms locatifs se distinguent des substantifs, des adverbes et des postpositions par l'opposition formelle du «cas commun » $(\mathrm{CMM})$ et des « cas obliques » (OBL). Les formes des cas obliques apparaissent là où le nom locatif se trouve à la fin d'un groupe nominal en position post-verbale (donc en fonction de complément d'objet indirect ou de circonstant), et la forme du cas commun est utilisée lorsque le nom locatif se trouve à la fin d'un groupe nominal occupant toute autre position syntaxique ${ }^{6}$ :

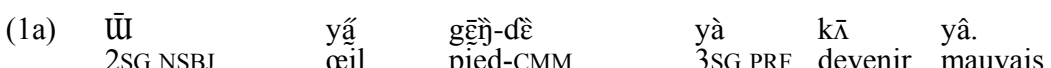
"Tu as de la saleté dans les yeux" (litt. : "le dessous de tes yeux est devenu mauvais"; position de sujet).

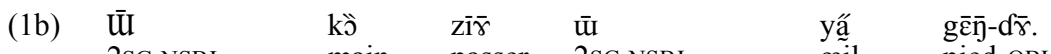
2SG.NSBJ main passer 2SG.NSBJ œil pied-OBL "Essuie sous tes yeux" (litt.: "Passe ta main sous tes yeux"; position de complément d'objet indirect).

Il faut préciser que les formes du cas commun peuvent apparaître en position de complément oblique avec une postposition (sans fusion!), là où la relation «nom-postposition » est moins usuelle :

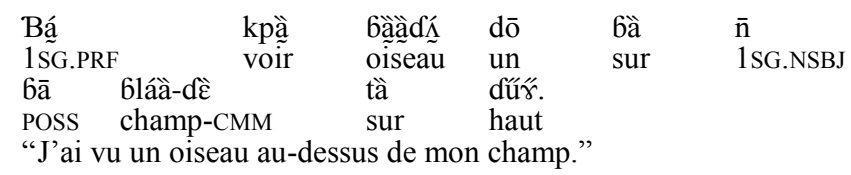

Au plan syntaxique, le nom locatif se distingue du substantif par sa capacité à apparaître en position de circonstant ou de complément d'objet indirect sans postposition. Il se distingue de la postposition par le fait qu'il peut (sous la forme d'un cas oblique) occuper seul cette position (ex. 3), tandis que la postposition doit obligatoirement avoir un groupe nominal à gauche (ex. $4 \mathrm{a}, \mathrm{b})$ :
(3) $\quad \mathrm{B} \ddot{\gamma}$

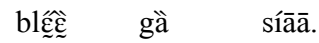

${ }^{5}$ Dans la version publiée du dictionnaire dan-gwèètaa (Vydrine, Mongnan 2008), il y en a 45 (sans compter de nombreux noms géographiques dans lesquels l'opposition morphologique «CMM : LOC » est très typique). Bien évidemment, ce nombre s'accroîtra dans la version finale.

6 Tous les exemples en dan-gwètaa sont présentés en transcription phonologique basée sur l'Alphabet Phonétique International (version pour l'Afrique). J'ai été obligé de lui donner la prépondérance dans cette étude, parce que l'orthographe officielle du dan de RCI ne permet pas de présenter correctement les tons dans les positions non-initiales. 
2SG.IMP 1SG.NSBJ ombre regarder terre.OBL

"Regarde mon ombre par terre."

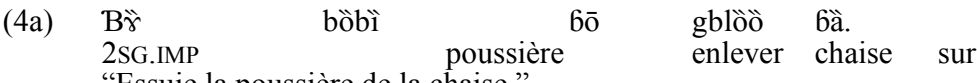

"Essuie la poussière de la chaise."

$\rightarrow(4 b) *$ Br̆ bòbì bō bà.

Par contraste avec l'adverbe, le nom locatif (sous la forme d'un cas oblique) est capable d'avoir un nom dépendant à sa gauche (cf. l'exemple (1b)).

\section{LA MORPHOLOGIE DES NOMS LOCATIFS}

\subsection{L'inventaire des cas}

La question se pose de savoir si les noms locatifs ont un seul cas oblique (assez hétérogène du point de vue formel) opposé au cas commun, ou plusieurs.

La première solution s'avère vulnérable quand on se heurte aux noms locatifs dont deux ou trois formes différentes (parfois même quatre) de cas obliques sont dérivés d'une même racine. En voici quelques-uns :

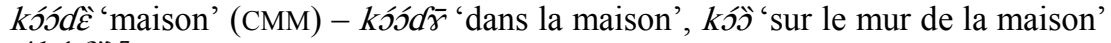
$(<* k j \text { bà })^{7}$

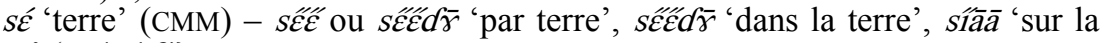
terre' $\left(<{ }^{*} S \varepsilon\right.$ É bä)

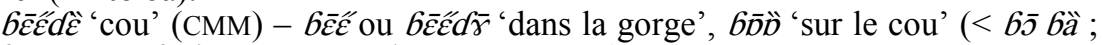
la forme non-fusionnée est également courante).

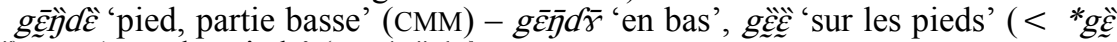
bä), $g \overline{\mathcal{E} \overline{\mathcal{E}}}$ 'avec les pieds' $\left(<{ }^{*} g \ddot{\mathcal{E}} k \hat{a}\right)$.

Voici les exemples illustrant l'emploi des trois cas obliques du pronom réciproque $k o ́$ :

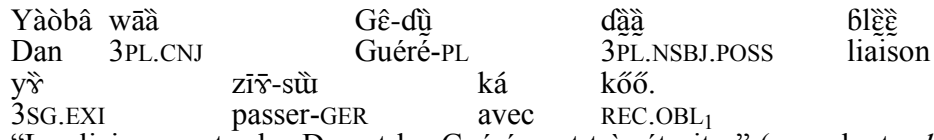

"Les liaisons entre les Dan et les Guéré sont très étroites" (sans doute, kôó < *kó-gúr).

(6a) Wồ tấ kî̀ wō

3PL.EXI danse fairelNEUT
kwn̂́ (sans doute, $<*$ kó-6ă).

7 Les formes non-fusionnées données entre parenthèses ont été produites par mon informateur principal qui se rendait compte, d'une façon plus ou moins certaine, du caractère dérivé des formes des cas obliques. 
REC.OBL 2

"Ils dansent ensemble."

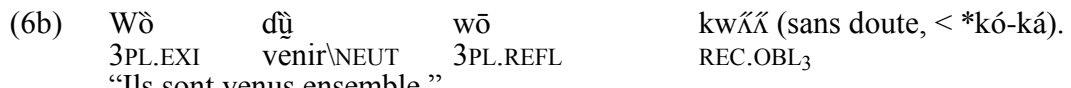

Dans certains contextes lexicalisés, ces formes servent à distinguer les valeurs habituelle et imperfective de la construction verbale de l'aspect neutre (sur l'aspect neutre en dan, cf. Vydrin 2010) :
(6c) Wò $\begin{array}{ll}\mathrm{y} \bar{\Lambda} & \mathrm{k} \dddot{\Lambda} \\ \text { travail } & \text { faire }\end{array}$

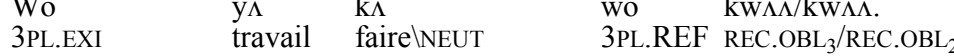 wō kwй̄̌/kwîñ. "Ils ont travaillé ensemble" / "Ils travaillent ensemble" (d'habitude).

En analysant les formes des noms locatifs, on peut distinguer :

a) le cas commun, le plus souvent marqué par le suffixe $-d \tilde{\varepsilon}$;

b) le cas locatif, le plus souvent marqué par le suffixe $-d \bar{r}$;

c) les autres cas obliques formés par la fusion avec quatre postpositions : gú 'dans', 6ä 'sur' (une surface non-plate), tä 'sur' (une superficie horizontale et plate), ká 'avec ; par'.

On pourrait donc parler d'un «cas commun» et de cinq cas «obliques»:

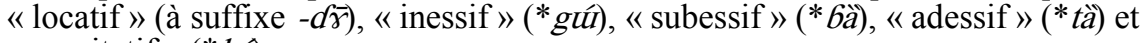
« comitatif » $(* k \hat{a})$.

Cependant, l'existence des cinq cas obliques doit être acceptée avec beaucoup de réserve, car le paradigme est loin d'être régulier. Il n'y a aucun mot qui aurait les six cas, et même ceux qui ont trois formes de cas (le cas commun plus deux cas obliques) sont peu nombreux. Le cas en dan-gwèètaa est une catégorie naissante, dont témoigne la facilité avec laquelle les locuteurs segmentent la plupart des formes en identifiant leurs composantes historiques. Dans la théorie grammaticale, on utilise pour les cas irréguliers de ce type le terme de «cas incomplet », ou de « cas partiel $»^{8}$.

Analysons les cas du nom locatif un par un.

\subsubsection{Le cas commun}

Du point de vue du sens grammatical, ce cas peut être considéré comme nonmarqué : il apparaît dans différentes positions syntaxiques, les mêmes que le nom; il ne se distingue que par l'opposition aux cas obliques. Du point de vue morphologique, il peut se présenter de deux façons, comme non-marqué (tó

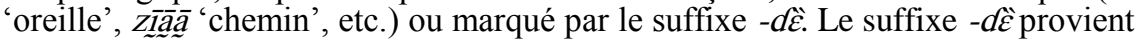
sans doute du substantif $d \widehat{\varepsilon}$ ' endroit'.

\footnotetext{
8 « Le cas incomplet est un cas dont les marques morphologiques n'apparaissent qu'avec une petite minorité des mots; pour le reste, les marques du rôle sémantique en question ne se distinguent pas des marques d'un autre cas » (Plungian 2000:175).
} 
Il faut mentionner que de nombreux noms locatifs à suffixe $-d \hat{\varepsilon}$ ont des substantifs corrélatifs de même racine : kóódê - kó 'maison', zūúúdè 'derrière' $z \bar{u}$ 'origine, base'. Le sémantisme des noms locatifs se distingue des substantifs de façon plus ou moins manifeste : kóx́dê désigne plutôt l'espace à l'intérieur de la maison, et $k o ́$ se dit lorsqu'on parle d'une maison comme bâtiment; zūúúdê a un sens concret (une partie du corps), et $z \bar{u} u$ a un sens abstrait ; $b \bar{\varepsilon} \varepsilon \tilde{\varepsilon} d \tilde{\varepsilon}$ désigne la partie antérieure du cou ou de la gorge, et $\bar{b} \bar{s}$ se réfère au cou en général et à la partie arrière du cou en particulier.

Dans d'autres cas, la différence sémantique entre les deux formes est assez difficile à déceler, mais elles se distinguent par leur emploi. Ainsi, dans la phrase (7a), aussi bien le substantif que le nom locatif sont possibles, tandis qu'en (7b), seul le substantif est admissible, et dans (7c), seul le nom locatif :

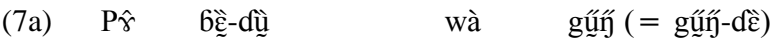
village humain $\backslash$ IZF-PL $\quad 3$ PL.PRF $\quad$ case.sacrée ( = case.sacrée-CMM)
dō dō.
certain mettre
"Les habitants du village ont bâti une case sacrée."

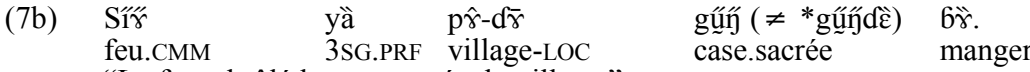
"Le feu a brûlé la case sacrée du village."
(7c) Gōō-gbêè-mè-nù wà̀ gwì zù̀̆ tête.dans-difficile-humain $\backslash$ IZF-PL $\quad 3$ PL.PRF pierre jeter

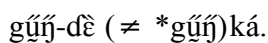
cáse.sacrée-CMM avec
"Les voyous ont jeté des pierres sur la case sacrée."

La comparaison des formes des noms locatifs et des substantifs correspondants laisse supposer qu'à part le suffixe - $-\hat{\varepsilon}$, il y a eu encore une composante, tantôt à ton haut, tantôt à ton extra-bas, qui s'est intercalée au milieu de la forme du nom locatif. Selon l'intuition de mon informateur principal, dans les exemples

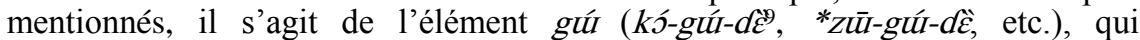
fonctionne en dan-gwèètaa comme un nom ('l'intérieur') et comme une postposition ('dans, en'). Et dans d'autres cas, il peut s'agir d'autres éléments à valeur locative. Considérons les formes des noms locatifs (cas commun) suivants, en comparaison avec les substantifs corrélés :

\begin{tabular}{|c|c|c|}
\hline & Nom locatif, CMM & Substantif \\
\hline 'paillote' & $g \overline{\mathcal{D}} \dddot{\eta} d \grave{\varepsilon}$ & $g \dddot{\jmath}$ \\
\hline 'jambes, pieds' & $g \bar{\varepsilon} \ddot{\eta} d \widehat{\varepsilon}$ & $g \ddot{\varepsilon}$ \\
\hline 'rocher plat' & $g_{W a ̀ a ̆ d \widehat{\varepsilon}}$ & $g_{W}$ àà \\
\hline 'côté' & $s \hat{\varepsilon} \hat{\tilde{j}} d \hat{\varepsilon}$ & $S \widetilde{\varepsilon}$ \\
\hline
\end{tabular}

${ }^{9}$ La forme kógúdề existe toujours comme une variante facultative de kóádê. 
Si tous les noms locatifs comportaient, à l'origine, la postposition gú, on ne s'attendrait pas à un ton extra-bas sur l'élement qui précède le suffixe -dêع. Il est fort probable que dans ce cas, l'élément précédant le suffixe - $d \widehat{\varepsilon}$ remonte à la deuxième postposition la plus courante du dan-gwè̀ta, bä.

Sans doute certains noms locatifs sont-ils dérivés des substantifs corrélés par simple adjonction du suffixe $-d \hat{\varepsilon}$, sans autres éléments, cf. :

\begin{tabular}{|c|c|c|}
\hline & Nom locatif, CMM & Substantif \\
\hline 'village' & $p \hat{\gamma} d \hat{\varepsilon}$ & $p \hat{\gamma}$ \\
\hline 'occiput' & kèëd है & kèè kèè \\
\hline
\end{tabular}

On trouve parfois des variantes phonétiques du même lexème au cas locatif qui se distinguent par leur syntaxe. Ainsi, les variantes du nom locatif pour 'ventre',

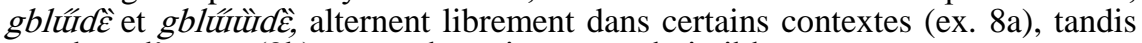
que dans d'autres (8b) une seule variante est admissible.

(8a) $\quad \mathrm{B} \bar{\varepsilon}$

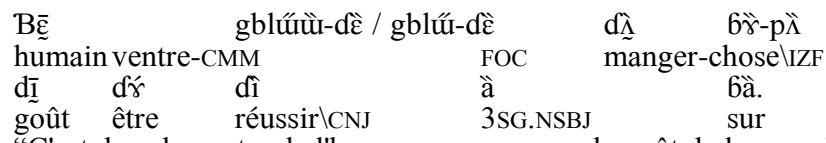

"C'est dans le ventre de l'homme que se passe le goût de la nourriture."

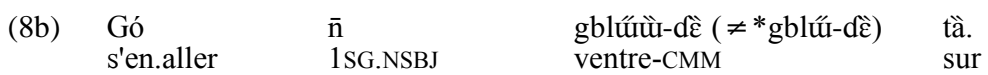

"Descends de mon ventre."

Apparemment, la forme gblúulùdề comporte historiquement une postposition (sans doute, bä) devant le suffixe $-d \hat{\varepsilon}$, tandis que la forme gblǘd̂̀ ne la comporte pas, ce qui a une incidence sur l'emploi des deux formes.

\subsubsection{Le cas locatif}

Normalement, la forme du cas locatif ne se distingue de la forme du cas

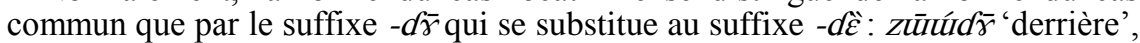

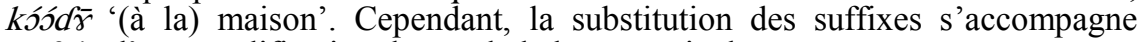
parfois d'une modification du ton de la base nominale :

$d \bar{\varepsilon} \bar{y} d \hat{\varepsilon}-d \bar{\varepsilon} \bar{y} d \bar{\gamma}$ 'champ',

$g \bar{\varepsilon} \bar{y} d \hat{\varepsilon}-g \bar{\varepsilon} \bar{\eta} \bar{\eta} d \bar{r}$ 'jambes, pieds',

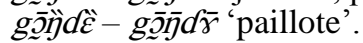

Il s'agit sans doute d'une assimilation tonale régressive dont la règle peut être formulée comme suit : " Si la base à structure $\mathrm{CVy}$ porte les tons moyen - extrabas dans le cas commun, le ton extra-bas est assimilé par le ton moyen du suffixe $-d \bar{\gamma}$ du cas locatif ». Facultativement, cette règle peut aussi s'appliquer aux bases 


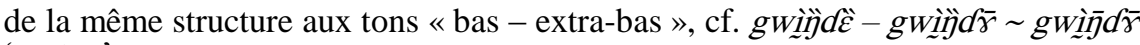
'vertex'.

Le suffixe $-d \bar{\gamma}$ peut être rapproché du suffixe homonyme $-d \bar{\gamma}$ qui sert à dériver les adverbes. On peut même considérer qu'il s'agit de deux emplois du même suffixe $-d \bar{\gamma}$, compte tenu de l'affinité fonctionnelle entre les adverbes et le cas locatif des noms locatifs ${ }^{10}$

Comme pour la forme du cas commun, la question se pose de savoir s'il y a eu historiquement un élément à valeur locative devant le suffixe $-d \bar{\gamma}$. On peut répondre avec assurance par l'affirmative, selon la même logique que dans 2.1.1. De plus, pour le cas locatif nous avons un test permettant d'établir à quelle postposition correspond le cas locatif : pour focaliser un nom locatif, il doit être mis au cas commun et frontalisé. Dans ce cas, la postposition réapparaît, précédée d'un pronom de reprise. Il s'avère que dans le corpus dont je dispose, c'est la postposition bä qui réapparaît, par exemple :

\begin{tabular}{|c|c|c|c|c|c|c|}
\hline (9a) & $\begin{array}{l}\text { Wà̀ } \\
\text { 3PL.PRF } \\
\text { "Elles ont étalé le }\end{array}$ & $\begin{array}{l}\text { s̄̄ } \\
\text { tissu } \\
\text { es vête }\end{array}$ & $\begin{array}{l}\text { kpó } \\
\text { étaler } \\
\text { nts sur u }\end{array}$ & \multicolumn{2}{|c|}{$\begin{array}{l}\text { gwàà-d的. } \\
\text { rocher.plat-LOC } \\
\text { rocher plat." }\end{array}$} & \\
\hline (9b) & $\begin{array}{l}\text { Gwàà-d̄̃ } \\
\text { rocher.plat-CMM } \\
\text { à } \\
\text { 3SG.NSBJ } \\
\text { "CC'est sur un roc }\end{array}$ & $\begin{array}{l}\text { d’̃ } \\
\text { FÖC } \\
\text { bä. } \\
\text { sur } \\
\text { her pla }\end{array}$ & $\begin{array}{l}\text { wà̀ } \\
\text { 3PL.PRF } \\
\text { u'elles c }\end{array}$ & t étalé le & vêtemen & $\begin{array}{l}\text { kpó } \\
\text { étaler }\end{array}$ \\
\hline (10a) & $\begin{array}{l}\text { Yà } \\
\text { 3SG.PRF } \\
\text { "Elle porte le bag }\end{array}$ & $\begin{array}{l}\text { kwè̌̀ } \\
\text { gage su }\end{array}$ & $\begin{array}{l}\text { sú } \\
\text { charge } \\
\text { a tête." }\end{array}$ & $\begin{array}{l}\bar{\gamma} \\
\text { prendre }\end{array}$ & 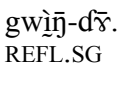 & sommet-LOC \\
\hline (10b) & $\begin{array}{l}\text { Ä } \\
\text { 3SG.NSBJ } \\
\text { sū } \\
\text { prendrelCNJ }\end{array}$ & $\begin{array}{l}\text { gwì̀- } \\
\text { somm } \\
\text { à } \\
\text { 3SG.Ns }\end{array}$ & CMM & $\begin{array}{l}\mathrm{d} \tilde{\Lambda} \\
\text { FOC } \\
\text { Gầ. } \\
\text { sur }\end{array}$ & $\begin{array}{l}\dot{\gamma} \\
3 \text { SG.CNJ }\end{array}$ & $\begin{array}{l}\text { kwè̀े } \\
\text { charge }\end{array}$ \\
\hline
\end{tabular}

L'apparition de 6ä comme postposition remplaçant la forme du cas locatif peut paraître en contradiction avec la fonction de gú comme « postposition par défaut" lors de la formation du pluriel des formes du cas locatif (cf. 2.2). D'autre part, La convergence des deux postpositions les plus courantes dans le

10 Cf. une observation de Denis Creissels (2009 : 613): "In languages in which nouns have a case inflection including a subsystem of spatial cases, spatial adverbs and adpositions commonly have possibilities of variation similar to noun case inflection, but limited to spatial cases. This can be viewed as an indication that, diachronically, such adverbs or postpositions originate from nominal forms inflected for case". En dangwèètaa, la dérivation des adverbes en $-d \bar{\gamma}$ semble être un procédé plus régulier que la formation des noms locatifs, il est donc plus approprié de penser que ce suffixe est d'origine adverbiale. 
cas locatif signale la rupture du lien étroit entre cas et postposition. Ce fait doit être interprété comme significatif d'un degré élevé de grammaticalisation du cas locatif.

Pour certains noms locatifs, le suffixe $-d \bar{\gamma}$ au cas locatif est facultatif :

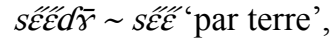

tààd $\bar{\gamma} \sim$ tàà 'au dos'

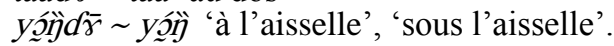

Cependant, cette situation peut recevoir une autre interprétation : les variantes asuffixales peuvent être considérées comme des formes d'autres cas obliques (subessif, inessif, etc.), malgré leur variation libre avec les formes du cas locatif dans certains contextes.

Dans un cas, la variante sans suffixe $-d \bar{\gamma}$ provient clairement d'une contraction de la forme régulière :

$p \hat{\gamma} d \bar{\gamma}^{\prime} \sim p l \bar{\gamma}^{-} \bar{\gamma}$ 'au village'.

Certains noms locatifs n'ont pas de suffixes casuels, que ce soit au cas commun ou au locatif :

bớCMM, bơ LOC 'initiation', 'lieu d'initiation',

bứ, blû́ CMM, bứ, blứ LOC 'brousse',

dí CMM, dí LOC 'bouche'.

Le cas locatif est le plus régulier des cas obliques (plus des trois-quarts de tous les noms locatifs), ce qui s'explique sans doute par sa valeur grammaticale assez large de « localisation non-spécifiée ».

\subsubsection{Le cas inessif}

Le cas inessif est rare; il n'a été relevé dans notre liste qu'avec neuf noms locatifs (cf. tableau en annexe). On observe parfois une alternance libre entre les formes des cas locatif et inessif, cf. (11), qui n'est pas possible dans bien d'autres cas $(12,13)$ :
(11) Yúờ

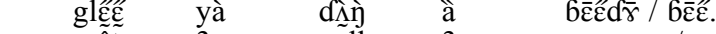
poisson arễte 3SG.PRF coller 3SG.NSBJ cou.LOC / cou.IN
"L'arête de poisson l'a étranglé” (litt.: “... s'est collé dans sa gorge”).
(12) Yà̀ à kú $\quad$ à $\bar{c} \bar{\varepsilon}(\neq * 6 \bar{\varepsilon} \bar{\varepsilon} d \bar{\gamma})$.

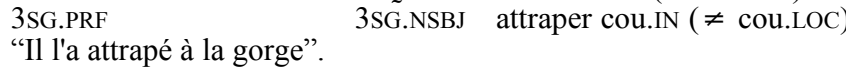

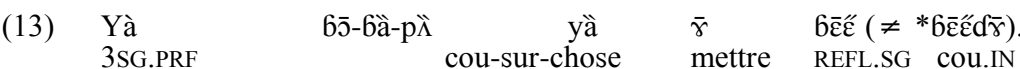
"Elle a mis un collier à son cou." 
L'affinité sémantique et fonctionnelle entre les cas locatif et inessif s'explique sans doute par le fait que de nombreuses formes du cas locatif comportent étymologiquement la postposition gú (la même dont provient le cas inessif).

\subsubsection{Le cas subessif}

Ce cas suit, par la fréquence, le cas locatif, il est signalé pour environ un quart des noms locatifs. L'analyse des formes subessives revèle qu'elles sont dérivées directement des substantifs, et non des formes du cas commun en $-d \hat{\varepsilon}$.

On peut établir des règles (ou, plutôt, des tendances) concernant la formation du cas subessif. Les substantifs (ou des noms locatifs au cas commun sans $-d \grave{\varepsilon}$ ) dont les formes subessives sont dérivées sont du type CV (et jamais CVV ou $\mathrm{CV}$ ). Le subessif se dérive le plus souvent par redoublement de la voyelle finale

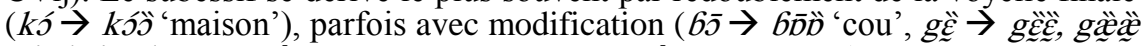

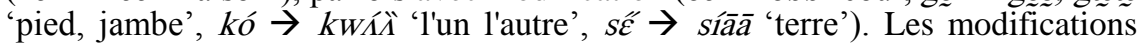
suivent presque toujours les modèles typiques de la fusion impliquant un morphème vocalisé en $-a$ en dan-gwèètaa. On observe cependant une modification plutôt atypique : $k \grave{\jmath} \rightarrow k w \varepsilon \grave{z}$ 'main' ${ }^{11}$. Dans tous les cas, le ton sur la voyelle finale est abaissé. Le plus souvent, il est extra-bas ; dans un seul cas, il est moyen (síāa 'terre', cf. la forme de la base de départ, sč́).

\subsubsection{Le cas adessif}

Les formes de ce cas, très marginal, n'ont été relevées que pour deux mots, $k j$

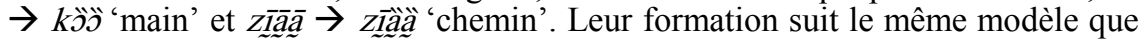
celle du cas subessif ; la forme seule ne suffit pas à déterminer de quel cas il

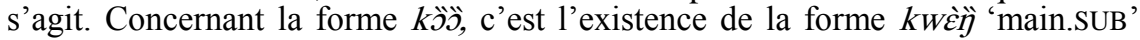
qui nous convainc que son cas n'est pas le subessif, et pour ziaäă, c'est l'existence de la forme de pluriel, zīââtädê-nù gúu, où l'élément d'origine postpositionnelle apparaît sans fusion.

\subsubsection{Le cas comitatif}

La postposition ká qui est à l'origine de ce cas a une polysémie très typique des langues mandé : elle exprime les valeurs comitative, instrumentale et équative, mais aussi (ce qui est moins courant dans les autres langues mandé) les valeurs de localisation spatiale ou temporelle générale. En parlant de «cas comitatif », je désigne l'ensemble de ces valeurs.

Le cas comitatif est assez rare : il n'est signalé que pour six noms locatifs. Les formes de ce cas sont dérivées selon la règle suivante :

1) la base de départ a obligatoirement la structure $\mathrm{CV}$;

2) la voyelle de la base de départ est redoublée, $\mathrm{CV} \rightarrow \mathrm{CVV}$;

${ }^{11}$ Sans doute, cette forme irrégulière provient de la dissimilation avec la forme du cas adessif. 
3) les tons des deux voyelles de la forme comitative sont uniformes: moyens, si le ton de la base de départ est extra-bas ( $k \ddot{\jmath} \rightarrow k \bar{\jmath} \bar{\jmath}$ 'main', $g \ddot{\mathcal{E}} \rightarrow g \overline{\mathcal{E}} \overline{\mathcal{E}}$ 'jambe, pied') ; extra-hauts, dans tous les autres cas.

Notons l'aptitude de la forme comitative du mot yấ 'œil' à adjoindre facultativement (dans certains contextes seulement) la postposition comitative :

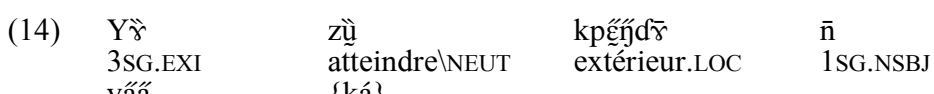

$$
\begin{aligned}
& \begin{array}{ll}
\text { yă̋å } & \{\text { ká\}. } \\
\text { yeux.com } & \text { avec } \\
\text { "Il est sorti en ma présence" (litt.: "avec mes yeux"). }
\end{array}
\end{aligned}
$$

Cette récurrence de la postposition comitative semble être un phénomène isolé.

\subsection{Le cas et le nombre}

Le pluriel du nom locatif au cas commun se forme par une simple addition du clitique -dù (comme pour les substantifs).

La formation du pluriel du cas locatif se réalise comme suit : la marque du cas commun - $d \grave{\varepsilon}$ s'ajoute à la suite du suffixe du cas locatif $-d(\bar{\gamma}$, et le clitique - $d \hat{u}$ suit le premier. Cette forme perd la capacité à remplir la fonction de complément d'objet indirect ou de circonstant sans postposition, elle est obligatoirement suivie de la postposition gú :

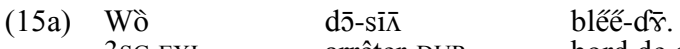
3SG.EXI arrêter-DUR bord.de.champ-LOC
"Ils sont arrêtés au bord du champ." $\rightarrow$

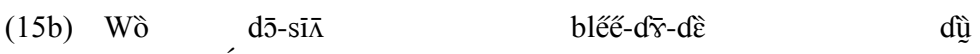 3SG.EXI ${ }^{\text {gú. }}$ arrêter-DUR bord.de.champ-LOC-CMM PL dans "Ils sont arrêtés aux bords des champs."

On trouve parfois des formes avec l'une ou l'autre marque casuelle omise ; ces

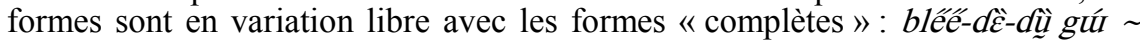

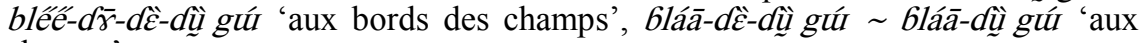
champs'.

Dans les autres cas obliques, les postpositions fusionnées tendent à réapparaître. Pour le reste, la formation de leur pluriel est identique :

(16a) Gō-dừ wồ zīàà

voiture-PL 3PL.EXI route.AD

"Des voitures sont sur la route." $\rightarrow$

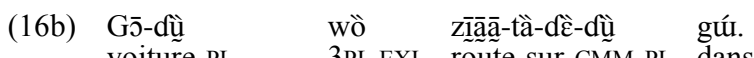
voiture-PL 3PL.EXI route-sur-CMM-PL dans "Des voitures sont sur les routes." 
Il faut noter que dans la pratique langagière, l'emploi des formes de pluriel des cas obliques est peu courante : selon la tendance générale en dan, le pluriel n'est employé que dans les contextes distinctifs, et dans les contextes de neutralisation de l'opposition du nombre on préfère les formes de singulier; cela concerne le plus souvent les cas obliques. Pour certains noms locatifs, le pluriel n'est pas

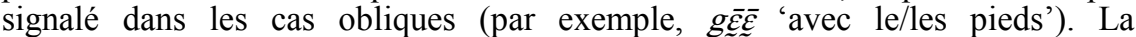
suppression de l'opposition du nombre dans les cas obliques ne représente rien d'extraordinaire du point de vue typologique (Plungian 2000).

\subsection{Les types morphologiques des noms locatifs}

On peut dire que le système de déclinaison des noms locatifs, du point de vue de la morphologie, est composé du noyau (opposition binaire quasi-régulière du cas commun en $-d \hat{\varepsilon}$ et du cas locatif en $-d(\vec{\gamma})$ et de la périphérie (les autres cas obliques, mais aussi les formes des cas commun et locatif sans suffixes). Dans le segment central, le cas commun, non-marqué du point de vue du sens grammatical, est cependant marqué formellement par le suffixe - $d \hat{\varepsilon}$ et différent du substantif corrélatif. Dans le segment périphérique, la forme du cas commun du nom locatif est non-marquée, et il est difficile, sinon impossible, de la distinguer du substantif corrélatif.

En fait, la limite entre les types du « noyau » et de la «périphérie » n'est pas nette, il y a des cas intermédiaires et hybrides. En simplifiant la situation, on pourrait parler de quatre types morphologiques de noms locatifs.

1) Les noms locatifs réguliers et quasi-réguliers : cas commun en - $d \widehat{\varepsilon}$ et cas locatif en $-d \bar{\gamma}$; certains lexèmes de ce type ont d'autres formes à d'autres cas (dérivées des substantifs corrélatifs).

2) Les noms locatifs invariables ont la même forme pour les deux cas, commun et locatif. Ils se différencient cependant des substantifs par un critère syntaxique, à savoir leur capacité à être employés en position post-verbale sans postposition :

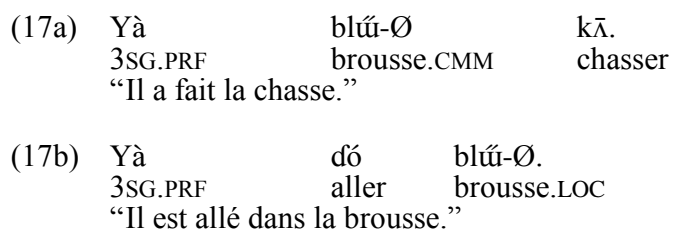

Ce type est représenté surtout par les toponymes ; il est peu fréquent parmi les noms communs.

Un nom locatif (17b) peut être homonyme du substantif correspondant (17c); dans ce cas, ils ne se distinguent que par l'emploi syntaxique en position post- 
verbale (avec ou sans postposition), tandis que dans les autres positions, le nom locatif et le substantif correspondant ne se distinguent pas :

(17c) Yà $\begin{aligned} & \text { Yó } \\ & \text { 3SG.PRF blú }\end{aligned}$ aller búl.
"Il est allé dans la brousse."

3) Les noms locatifs asuffixaux ont la même racine que la forme du cas commun, et les formes des cas obliques en dérivent par des modifications tonales et/ou vocaliques. Il s'agit surtout des cas obliques autres que le locatif (cf. 2.1.3. - 2.1.6.). Dans certains cas, les locuteurs du dan-gwèètaa acceptent également les formes non-fusionnées en les qualifiant « de formes du langage enfantin » (zīāa tà $=$ Zīâà, $s \tilde{\varepsilon}$ bà $=$ sîââ).

4) Les noms locatifs «mixtes» combinent des caractéristiques des types régulier et invariable : la forme du cas commun coïncide avec la racine (au moins

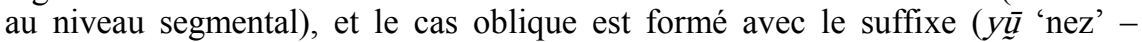

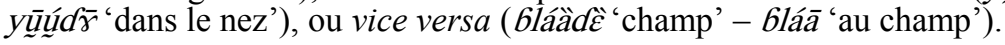

\section{LA SYNTAXE DES CAS}

Les cas obliques en dan-gwèètaa restent étroitement liés aux postpositions dont ils proviennent, et l'emploi des formes suit grosso modo les modèles d'emploi des postpositions en question (cf. cependant la division 4 où certains modèles plus compliqués de distribution des cas et des postpositions sont mentionnés).

Considérons quelques particularités d'emploi des noms locatifs qui ne semblent pas tout à fait prévisibles.

\subsection{Le groupe nominal possessif : la concordance des cas?}

Là où un syntagme possessif se trouve dans la position du circonstant ou du complément d'objet indirect, la marque possessive sert d'indicateur supplémentaire du cas oblique. Dans le cas général, le connecteur possessif est bà $(18 \mathrm{a}, 19 \mathrm{a})$, mais là où la tête du syntagme possessif est représentée par un nom locatif au cas locatif, ce connecteur est remplacé par gö $(18 \mathrm{~b}, 19 \mathrm{~b})$ :

\begin{tabular}{|c|c|c|c|c|c|}
\hline (18a) & $\begin{array}{l}\overline{\mathrm{N}} \\
\text { 1SG.NSBJ } \\
\text { tồ } \\
\text { resterlNEUT } \\
\text { "La limite }\end{array}$ & $\begin{array}{l}\text { 6ā } \\
\text { POSS } \\
\text { yígā } \\
\text { rivière } \\
\text { n cham }\end{array}$ & $\begin{array}{l}\text { 6láà-d̋̀ } \\
\text { champ-CMM } \\
\text { bầ. } \\
\text { sur } \\
\text { oasse par la ri }\end{array}$ & $\begin{array}{l}\text { d'́r" } \\
\text { limite }\end{array}$ & $\begin{array}{l}\text { y } \\
\text { 3sG.EXI }\end{array}$ \\
\hline$(18 b)$ & $\begin{array}{l}\text { Bá } \\
1 \text { SGG.PRF } \\
\text { 6láā } \\
\text { champ.LOC }\end{array}$ & $\begin{array}{l}\text { dó } \\
\text { aller } \\
\text { būū. } \\
\text { là-bas }\end{array}$ & $\begin{array}{ll}\overline{\mathrm{n}} & \text { gò } \\
\text { 1 SG.NSBJ }\end{array}$ & & \\
\hline
\end{tabular}


"Je suis allé à mon champ."
(19a) $\begin{array}{lllll}\text { À } & \text { 6à } & \text { ḱ } & \text { y r̀ } & \text { g r̀ } \\ \text { 3SG.NSBJ } & \text { POSS } & \text { maison } & \text { 3SG.EXI } & \text { brûler|NEUT }\end{array}$ d"ع́d"̌́ plè ká.

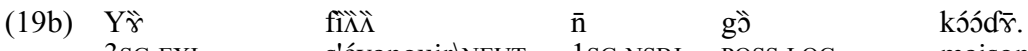
3SG.EXI s'évanouirlNEUT 1SG.NSBJ POSS.LOC maison.LOC
"Il s'est évanoui dans ma maison."

Il est difficile de résister à la tentation de voir là une concordance des cas (bà pour les substantifs et les noms locatifs au cas commun, gö pour les noms locatifs au cas locatif), ce qui signifierait un degré assez avancé de maturité du système des cas en dan-gwèètaa. Cependant, certaines données incitent à la réserve.

Tout d'abord, il semblerait que l'emploi du connecteur possessif locatif gö dans le contexte désigné soit préférable, mais facultatif; la marque bà y est également possible (cf. exemple (20)), même si elle y est beaucoup plus rare.

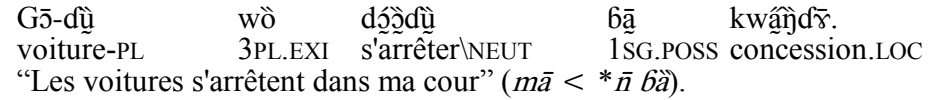

Ensuite, la marque possessive gö apparaît parfois dans le groupe nominal circonstanciel même si la possession est exprimée par un substantif (21) ou un nom locatif à d'autres cas obliques (22).

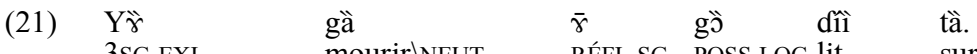
3SG.EXI mourir|NEUT RÉFL.SG POSS.LOC lit sur
"Il est mort dans son lit."

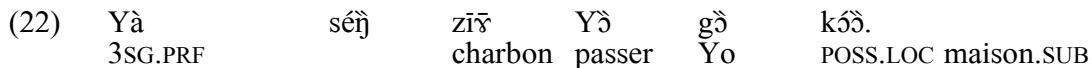
"Il a laissé des traces de charbon sur le mur de la maison de Yo."

Ces exemples montrent que l'emploi de gö est conditionné plutôt par la position syntaxique que par le cas du nom suivant. Cependant, on ne peut pas nier que dans la grande majorité des cas, cette marque cooccurre avec le cas locatif.

\subsection{Cas et détermination}

La présence d'un adjectif (placé en dan après le nom) bloque la déclinaison :
(23a) Wà

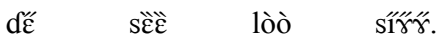
2PL.PRF feuille frais arriver feu.LOC

"On a mis des feuilles vertes dans le feu." 


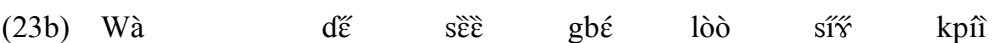 3PL.PRF feuille frais grand arriver feu.CMM grand

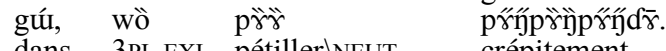 dans 3PL.EXI pétiller|NEUT crépitement "Lorsqu'on met beaucoup de feuilles vertes dans un grand feu, elles crépitent."

De même, le nom à un cas oblique ne peut pas être focalisé ou spécifié par un déterminant (pronom démonstratif, article). Là où un déterminant intervient, le nom locatif est remplacé par un substantif :
(24a) Bá-'

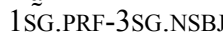
$\begin{array}{lll}\text { wó } & \mathrm{d} \overline{0} & \mathrm{~d} \hat{\varepsilon} \\ \text { affaire } & \text { savoir } & \text { que }\end{array}$
$6 \bar{\varepsilon} \quad y \dddot{\gamma}$
kóó-d $d \bar{\gamma}$.
personne 3SG.EXI
maison.LOC
"J'ai senti qu'il y avait quelqu'un dans la maison."

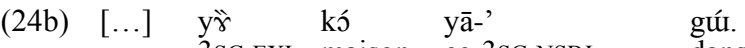 3SG.EXI maison ce-3SG.NSBJ
"[...] qu'il y a quelqu'un dans cette maison".
(25a) Y Ÿ

$\begin{array}{lll}\text { dồ } & \text { à } & \text { 6à̀-’. } \\ \text { allerlNEUT } & \text { 3SG.NSBJ } & \text { frapper-INF }\end{array}$

3SG.EXI monterlNEUT maison-LOC déjà 1SG.EXI
"S'il entrait dans la maison, je le frapperais."
(25b) $[\ldots] \quad k \bar{\gamma} \quad$ wò̀ dà $\quad$ kó bā-’ gú “[...] pour qu'ils entrent dans la maison'.

Cela veut dire que le système des cas en dan-gwèètaa est encore suffisamment jeune pour ne pas être détaché du contexte originel : comme une postposition suit obligatoirement le groupe nominal, une forme au cas oblique ne peut pas apparaître là où le nom locatif n'occupe pas la position finale dans un tel groupe.

Cependant, certaines formes isolées ont recours à une autre stratégie pour contourner cet obstacle : la fusion des noms avec un déterminant démonstratif et une postposition :

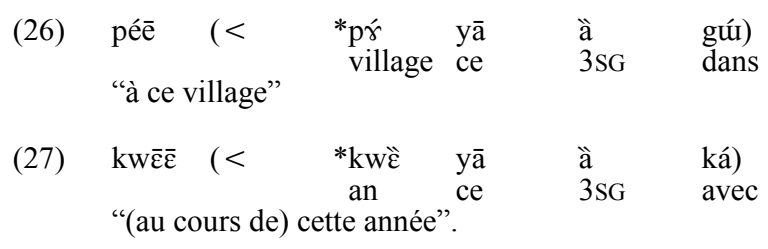


"Son couteau est dans sa main."

\begin{tabular}{|c|c|c|c|c|c|}
\hline (31b) & $\begin{array}{l}\text { Ä } \\
\text { 3SG.NSBJ } \\
\text { kwè̀̆ kwè̀̀. } \\
\text { main.SUB INT } \\
\text { "Son couteau e. }\end{array}$ & $\begin{array}{l}\text { bà } \\
\text { POSS }\end{array}$ & $\begin{array}{l}\text { dâà-d' }{ }_{\tilde{\tilde{n}}} \\
\text { couteâu-DIM }\end{array}$ & sa main." & à̀ \\
\hline (32a) & $\begin{array}{l}\text { Bā } \\
\text { 1 } \text { "M.POSS } \\
\text { "Mon chien est }\end{array}$ & 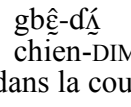 & & $\begin{array}{l}\text { yæ̆ } \\
\text { 3SG.EXI }\end{array}$ & 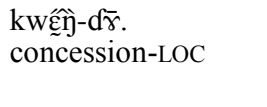 \\
\hline (32b) & $\begin{array}{l}\text { Bāa } \\
\text { 1 } \text { SG.POSS } \\
\text { yáá } \\
\text { 3sG.NÉG.IPFV }\end{array}$ & 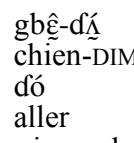 & place & $\begin{array}{l}\text { yð̆ } \\
\text { 3SG.EXI } \\
\text { 6á } \\
\text { certain }\end{array}$ & 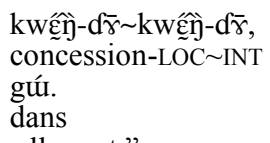 \\
\hline
\end{tabular}

Autrement dit, nous avons ici afaire à un marquage externe : la valeur d'une marque morphologique apparaissant sur un mot porte sur un tout autre mot $^{13}$.

\subsubsection{Quelques traits morphologiques et sémantiques du redoublement des noms locatifs}

Le redoublement des noms locatifs est un phénomène assez productif, mais il n'est pas systématique. Mon informateur a rejeté les formes redoublées de nombreux lexèmes, et pour certaines autres, il les a acceptées avec des réserves («ça se dit parfois, mais ce n'est pas très joli, je ne le mettrais pas dans le dictionnaire »).

Les formes de la majorité des cas obliques sont susceptibles d'être redoublées ; je n'ai pas trouvé d'exemples de cas comitatif et adessif, les plus marginaux dans le système de la déclinaison dan-gwè̀taa.

Le plus souvent, le redoublement est complet. Cependant, on trouve des cas $(33 \mathrm{~b}, 34 \mathrm{~b})$ où la forme redoublée du cas locatif perd sa désinence $-d \bar{\gamma}$ :
(33a) Siăô yö̀ kóó- $d \bar{\gamma}$.
NOM 3SG.EXI maison-LOC
"Siao est à la maison."

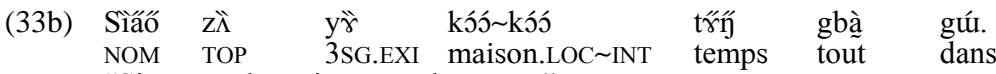 "Siao est à la maison tout le temps."

13 Le dan-gwèètaa est très riche en marquages externes : le pluriel des noms peut être exprimé sur les adjectifs (Vydrine 2007) ; les déterminants attachés à un préverbe portent sur le verbe (Vydrin 2009), etc. Ce phénomène semble être une tendance prédominante de cette langue. 


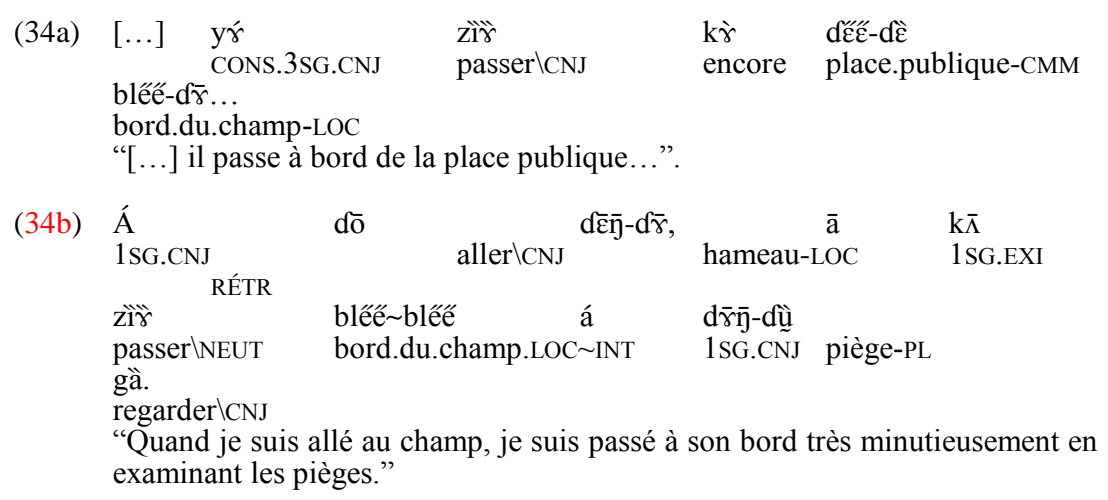

Du point de vue du sens, le redoublement des noms locatifs exprime le plus souvent l'insistance sur le caractère permanent et prolongé de l'action ou de la situation $(31 \mathrm{~b}, 32 \mathrm{~b}, 33 \mathrm{~b})$, mais il peut s'agir également d'une action détaillée et exhaustive (34b). De fois, le redoublement produit une modification de sens tout à fait irrégulière, comme dans $(35 \mathrm{a}, \mathrm{b})$ :

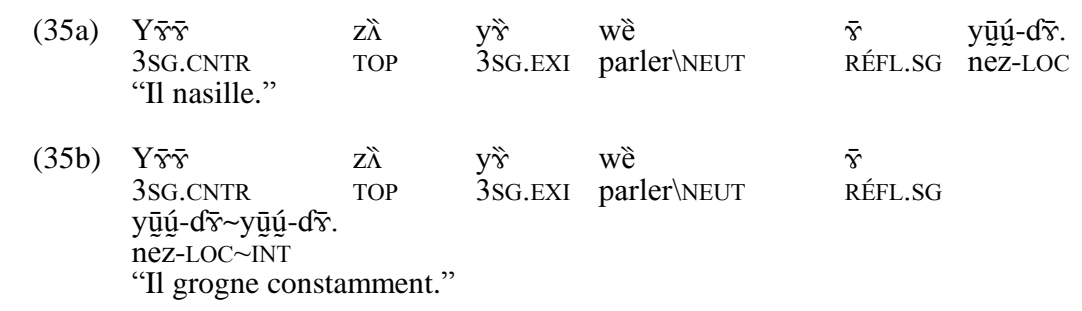

\subsubsection{Du redoublement des noms locatifs au redoublement des postpositions}

Le redoublement des noms locatifs en dan-gwèètaa n'est pas un phénomène isolé : certaines postpositions peuvent être redoublées également.

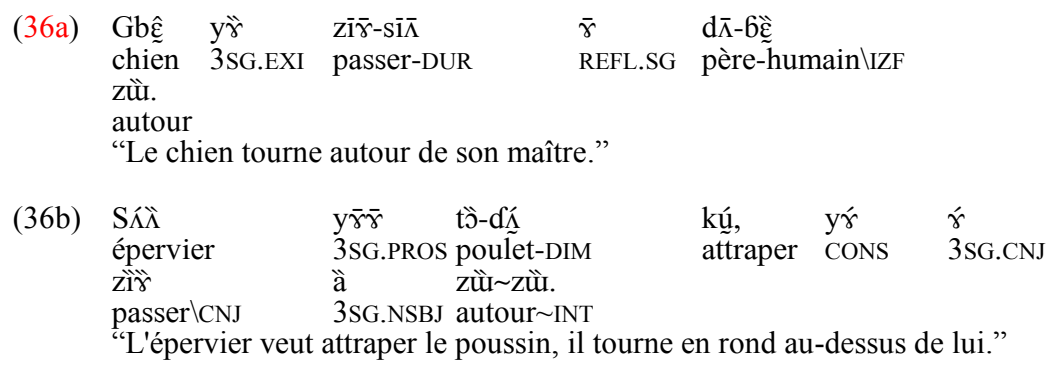




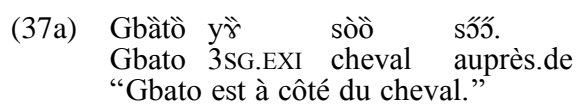

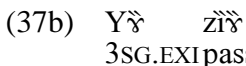
3SG.EXIpasserlNEUT bَ̃ -dü.

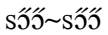
gbé.
"Il tourne trop autour des gens."
auprès.de INT
beaucoup

Comme le montrent ces exemples, le redoublement des postpositions représente le même type de marquage extérieur à valeur d'intensité, de caractère intensif ou prolongé de l'action exprimée par le verbe. Une autre caractéristique est le caractère sélectif du redoublement des postpositions : à ma connaissance, il ne concerne que quatre postpositions à valeur locative (sóś' 'auprès de', zǜ 'autour de', kèn̈ 'derrière', pḯr 'chez'). On retrouve ici l'affinité entre noms locatifs et postpositions. En fait, il n'est pas facile parfois de décider si un lexème doit être considéré comme un nom locatif relationnel ou comme une postposition, cf. (38) :

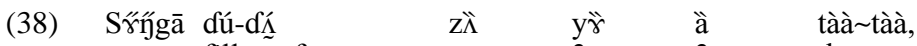
NOM fille-enfant TOP 3SG.EXI 3SG.NSBJ dos.LOC $\sim$ INT $\begin{array}{lll}\text { yáá } & \text { y } \bar{\gamma} & \text { síāā. } \\ \text { 3SG.NÉG.IPFV } & \text { voir } & \text { terre.SUB }\end{array}$
"La fille de Seunga est toujours sur son dos, elle ne descend pas par terre".

Dans cet exemple, la forme tààtàà peut être analysée soit comme un nom relationnel 'au dos', soit comme une postposition 'derrière'.

\subsubsection{Un cas intermédiaire : des postpositions et des adverbes composés?}

Considérons certains autres cas de redoublement post-verbal (ex. 39b, 40b, 41b, 42) :
(39a) $\mathrm{Y} \ddot{\mathrm{r}}$
3SG.EXI

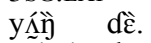
र bà kwèc ká
soleil devant
"Il passe avec sa charge au soleil."

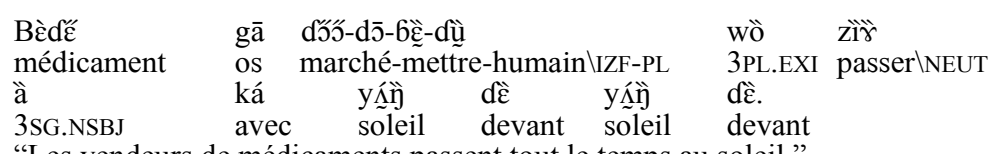
"Les vendeurs de médicaments passent tout le temps au soleil."

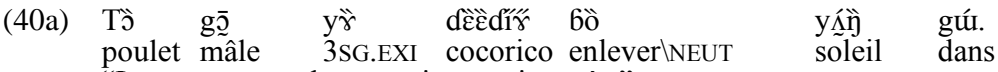 "Le coq pousse des cocoricos en journée."
(40b) Tə
ḡ $\quad y \ddot{\gamma}$

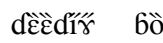
y수ำ gú 
poulet mâle 3SG.EXI cocorico enleverlNEUT soleil dans

yরূî̀ gú.

soleil dans

"Le coq pousse des cocoricos toute la journée."

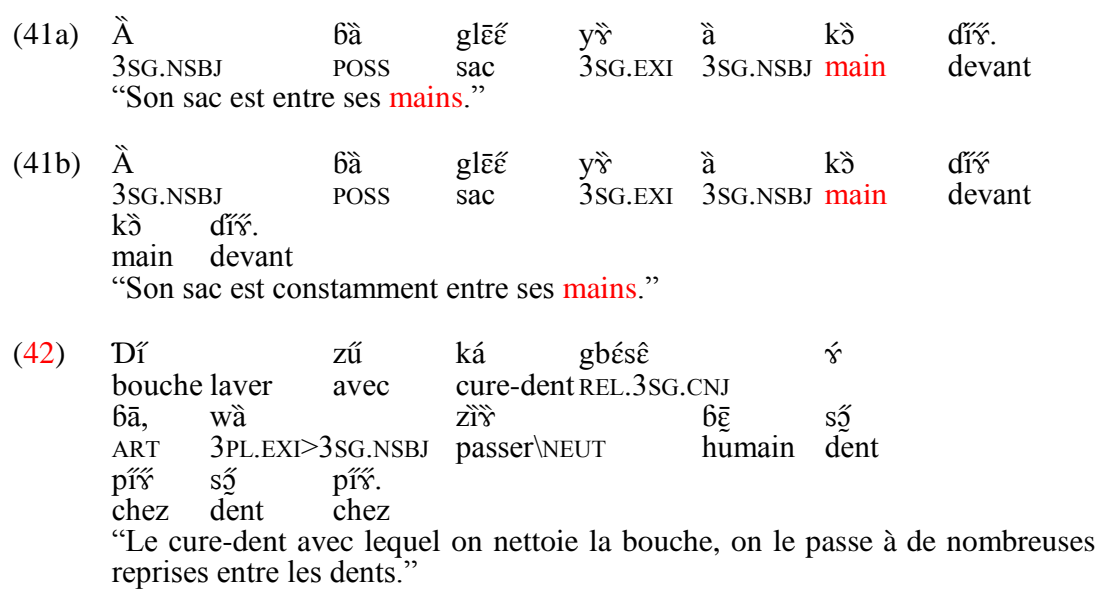

De toute évidence, nous avons ici le même phénomène de redoublement d'un élément post-verbal en fonction de marquage externe exprimant l'intensité ou le caractère permanent de l'action. La question se pose de savoir quel statut

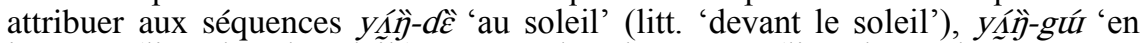

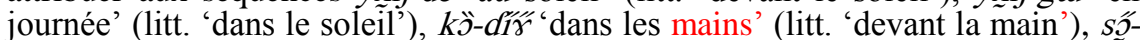
píz' ‘entre les dents' (litt. : 'chez les dents') et autres expressions redoublées de ce type. Leur aptitude au redoublement est un argument sérieux pour les considérer comme des lexèmes uniques, plutôt que des combinaisons (libres ou idiomatisées) de paires de lexèmes ${ }^{14}$.

Il semble peu approprié de les mettre dans la classe des noms locatifs : on n'observe pas de fusion morphologique entre les bases nominales et postpositionnelles (ce qui est typique des vrais noms locatifs); certaines séquences, comme yấn̈-gú, comportent les mêmes postpositions qui sont déjà intégrées dans les noms locatifs ; l'assimilation de ces formes aux noms locatifs conduirait à une inflation de cette classe préjudiciable à sa cohérence.

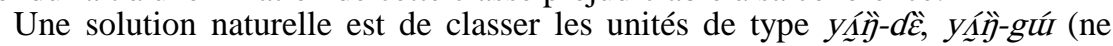
pouvant pas avoir un dépendant à gauche) parmi les adverbes, et les unités de type $k \grave{\text { - }}$ -

14 On peut mentionner, comme un précédent, le cas des langues turques où la capacité d'une unité au «redoublement en $m$-» est vue comme un argument suffisant pour considérer cette unité comme un lexème, plutôt que comme une combinaison de lexèmes (je remercie Daria Mishchenko pour cette observation). 
complétera par une sous-classe d'adverbes composés, et la classe des postpositions, par des postpostions composées.

\section{LIMITES SÉMANTIQUES DE LA CLASSE DES NOMS LOCATIFS}

Le processus de formation des noms locatifs en dan-gwètaa est très sélectif. Tout d'abord, les limites sémantiques de cette classe de lexèmes sont assez nettes : il s'agit (a) des noms des parties du corps, et (b) des mots communs désignant des «localisations naturelles », qui peuvent typiquement servir de localisation des actions ou des situations. Beaucoup de noms géographiques (surtout ceux qui sont traditionnellement connus des Dan) distinguent les formes

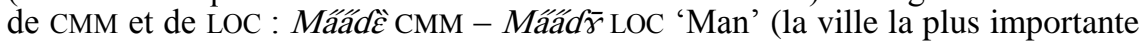

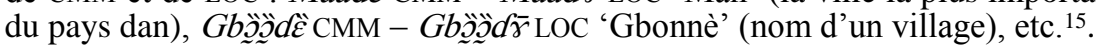

Ensuite, la fusion avec les postpositions se produit dans les contextes les plus typiques du lexème en question, tandis que dans d'autres contextes une fusion avec la même postposition peut ne pas avoir lieu. Ainsi, nous avons le cas comitatif (dans ce contexte, à valeur instrumentale) du mot š̃ 'dent' dans l'expression qui signifie « mordre » :
(43a) Yà $\begin{array}{lll}\text { Yà } & \bar{\gamma} & \text { dè } \\ \text { 3SG.PRF } & \text { REFL.SG } & \text { même }\end{array}$

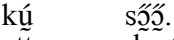
"Il a mordu soi-même."

Dans d'autres contextes, les mêmes composantes ne fusionnent pas :
(43b) Yà wlर́ $\quad$ sú $\quad \bar{\gamma} \quad$ š̃ 3SG.PRF mortier prendre REFL.SG dent avec "Il a soulevé le mortier avec ses dents."

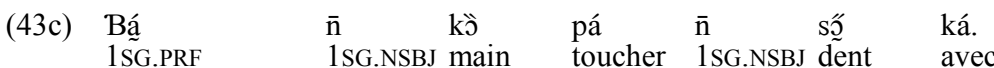 "J'ai touché mes dents."

Le mot polysémique $s \widehat{\varepsilon}$ 'sol, terre ; pays' peut apparaître au cas inessif dans son premier sens (44), mais pas dans le deuxième (45) :
$\begin{array}{lll}\text { Bāā } & \text { bā } & \text { à } \\ \text { manioc } & \text { ART } & \text { 3SG } \\ \text { "Enlève le manioc de la terre." }\end{array}$

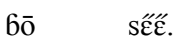
sortir terre.IN

\begin{tabular}{|c|c|c|c|}
\hline $\begin{array}{l}\text { Dëkpp̀òyî̀ } \\
\text { jour }\end{array}$ & 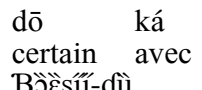 & $\begin{array}{l}\text { ph-dề } \\
\text { village-CMM } \\
\text { gô }\end{array}$ & $\begin{array}{l}\text { Gá } \\
\text { certain } \\
\text { gúl }\end{array}$ \\
\hline $\begin{array}{l}\dot{\gamma} \\
\text { REL.3SG.CNJ }\end{array}$ & $\begin{array}{l}\text { Bö̀ัšsíí-dù̀ } \\
\text { Bũrkinaîs-PL }\end{array}$ & $\begin{array}{l}\text { gò } \\
\text { POSS.OBL terre }\end{array}$ & $\begin{array}{l}\text { gúu... } \\
\text { dans }\end{array}$ \\
\hline
\end{tabular}

15 Les relations spéciales des noms de «localisations naturelles» et des noms géographiques avec les cas spatiaux ont été traitées par Denis Creissels (2009: 612-613). 
Pour désigner la maison, on a en (22) un nom locatif au cas subessif (provenant de la fusion avec la postposition 6ä), tandis qu'en (46) nous trouvons un substantif avec bä sans qu'une fusion n'intervienne :

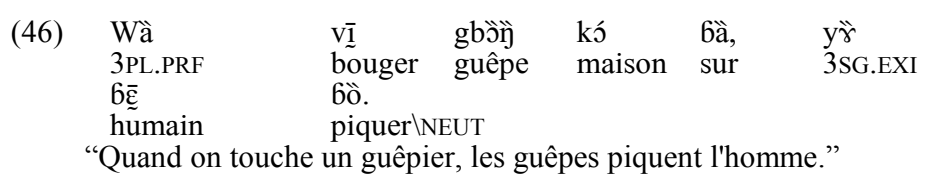

Les exemples $(47,48 \mathrm{a})$ (par contraste avec (13)) et $(48 \mathrm{a}, \mathrm{b})$ montrent que la fusion des postpositions gú et bä avec le substantif $6 \overline{5}$ 'cou' n'est pas du tout automatique et dépend du contexte :

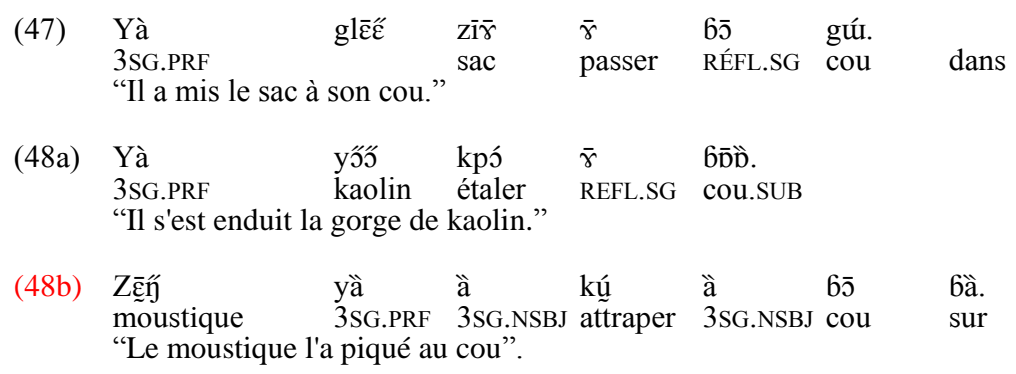

Le degré élevé de lexicalisation témoigne du fait que la morphologisation des cas en dan-gwèètaa est un processus assez avancé; on ne peut plus considérer une forme de cas oblique comme un équivalent facultatif d'une combinaison d'un substantif avec une postposition; il s'agit bien de formes flexionnelles avec leur propre sémantisme et leur syntaxe.

\section{QUELQUES MOTS CONCERNANT LES NOMS LOCATIFS DANS D'AUTRES LANGUES MANDÉ}

La tendance à la formation de «noms locatifs » est attestée dans la majorité des langues mandé-sud, mais dans chacune des langues elle a ses particularités. En beng, ils ne se distinguent des autres substantifs que syntaxiquement (par leur capacité à fonctionner comme circonstant ou complément d'objet indirect sans postposition) (Paperno 2011: 46-48). En mwan, gouro, kla-dan, kpelle, le nombre de noms locatifs dépasse à peine une douzaine (communications personnelles d'Elena Perekhvalskaya, Natalia Kuznetsova, Maria Konoshenko, Nadezda Makeeva) ; ils se distinguent morphologiquement des autres substantifs, mais leur statut reste à préciser. En toura, ils se distinguent morphologiquement des autres substantifs, mais, à la différence du dan, ils peuvent occuper les positions de sujet et de complément d'objet direct (Bearth 1971: 199-201). 
Autrement dit, le critère syntaxique ne permet pas de les distinguer des substantifs dans cette langue.

La fusion des noms avec les postpositions est signalée en gouro, mais dans cette langue tous les cas de fusion sont transparents et peuvent être remplacés synchroniquement par une combinaison «nom + postposition ». Autrement dit, ici la distinction entre "noms locatifs» et substantifs est assez faible (communication personnelle de Natalia Kuznetsova). Sans doute, la situation en yaouré est identique.

On peut parler de «noms locatifs » même dans les langues mandingue. Par exemple, en bambara la majorité des toponymes s'emploie en position de circonstant sans postposition, et cela est caractéristique également de quelques noms communs, en particulier dá 'bouche' et só 'maison' :

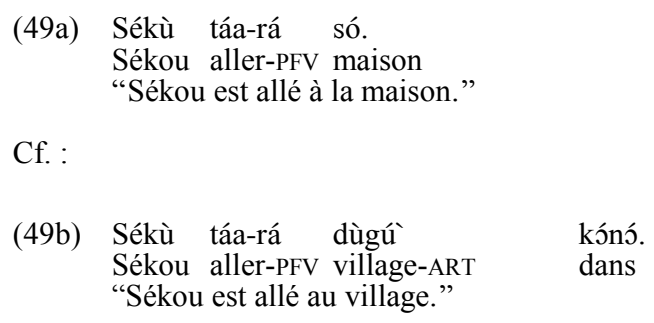

\section{CONCLUSION}

Le dan-gwèètaa fournit un exemple intéressant d'un système de déclinaison en formation, d'où ses caractéristiques :

- un nombre limité de lexèmes concernés, qu'on est donc obligé de considérer comme une sous-classe ( «les noms locatifs ») de la partie du discours du nom, et dont la limite avec les autres noms («les substantifs ») n’est pas toujours très nette ;

- le caractère aléatoire du paradigme de déclinaison, l'absence de noms locatifs ayant un paradigme complet ;

- la transparence étymologique des formes flexionnelles (malgré la technique de fusion) et la possibilité de rétablir, assez souvent, des formes originales par les locuteurs de la langue; réapparition de la structure morphologique originelle dans les formes plurielles (là où elles sont possibles).

L'apparition de la déclinaison en dan-gwèètaa, tout en étant un fait exceptionnel dans la famille mandé, est le prolongement de deux tendances connues des nombreuses langues apparentées :

1) utilisation des noms à valeur locative en fonction de circonstant sans postposition, ce qui les rapproche des adverbes ;

2) fusion, facultative ou obligatoire, des noms en position de circonstant avec les postpositions.

En dan-gwè̀taa, la première tendance a été compliquée par le fait que les adverbes se caractérisent dans cette langue, le plus souvent, par un suffixe 
dérivatif $-d \bar{\gamma}$. Le même suffixe a acquis une nouvelle fonction : marque de la fonction circonstantielle du nom.

Ce processus a été conforté par la deuxième tendance qui a produit des formes fusionnées des noms avec des postpositions. La fonction syntaxique des formes fusionnées est la même que celle des formes nominales en $-d \bar{\gamma}$.

Le système naissant de la déclinaison en dan a donc deux sources, l'un (les adpositions) étant très connu dans l'histoire de langues diverses (cf., en particulier, Kulikov 2006 : 23 ; Kulikov 2009 : 440-445), l'autre, l'intégration des adverbes dans le paradigme nominal de déclinaison, étant plus exotique, et cependant ayant des analogies (en particulier, en ossète) dans l'histoire des évolutions des systèmes des cas (Kulikov 2006 : 29 ; Kulikov 2009 : 445-447).

Le paradigme de déclinaison des noms locatifs en dan-gwèètaa représente un modèle peu fréquent où les rôles syntaxiques du noyau (exprimés dans d'autres systèmes par des «cas grammaticaux» (Blevins 2009: 200-201) ne se distinguent pas, ils sont tous marqués par le «cas commun ». L'opposition du «cas commun» au «cas locatif» se trouve au centre du système dont la périphérie est constituée par les autres cas obliques à valeur spatiale ou instrumentale/comitative.

Cependant, ce système n'est pas exceptionnel à l'échelle mondiale; des systèmes à cas locatifs où les arguments syntaxiques principaux ne sont pas distingués par les cas sont bien attestés. Ainsi, Creissels (2009:611) parle de ce type de paradigme dans les langues tswana et nahuatl.

\section{Abréviations}

$\mathrm{AD}-$ cas adessif

ART - article défini

CMM - cas commun

CNJ - marque prédicative pronominale de la série conjointe ; forme verbale dans la construction conjointe

CNTR - série des pronoms contrastifs

$\mathrm{COM}$ - cas comitatif

CONS - conjonction à valeur consécutive

DIM - suffixe diminutif

DUR - suffixe de duratif

EXI - marque prédicative pronominale de la série existentielle

FOC - déterminant focalisateur

GER - gérondif

IMP - marque prédicative pronominale de la série impérative

IN - cas inessif

INF - marque de l'infinitif

INT - intensif

IPFV - imperfectif

IZF - marque tonale de l'izafet (ton extra-bas sur le mot gouvernant)

LOC - cas locatif

NÉG - marque négative

NEUT - marque de l'aspect neutre (ton extra-bas suffixé au verbe)

NOM - nom propre 
NSBJ - pronom de la série non-sujette

OBL - oblique

OPT - marque prédicative de la série optative

$\mathrm{PFV}$ - marque du perfectif

PL - pluriel

POSS - marque possessive

PRF - marque prédicative pronominale de la série du parfait

PROS - marque prédicative de la série prospective

RÉC - réciproque

RÉFL - pronom réfléchi

$\mathrm{REL}$ - marque du relatif

RÉTR - marque rétrospective

SG - singulier

SUB - cas subessif

TOP - marque de topicalisation

\section{RÉFÉRENCES}

Bearth Th., 1971, L'énoncé toura, Norman (Oklahoma), S.I.L.

Blevins J., 2009, Case and declensional paradigms, in A. Malchukov \& A. Spenser (eds.), The Oxford handbook of case, Oxford University Press, p. $200-218$.

Creissels D., 2009, Spatial cases, in A. Malchukov \& A. Spenser (eds.), The Oxford handbook of case, Oxford University Press, p. 609-625.

Dimmendaal G., 2008, Language Ecology and Linguistic Diversity on the African Continent, Language and Linguistics Compass 2/5, p. 840-858.

König Ch., 2008, Case in Africa, Oxford and New York, Oxford University Press.

Kulikov L., 2006, Case systems in a diachronic perspective : A typological sketch, in L. Kulikov, A. Malchukov \& P. de Swart (eds.), Case, Valency and Transitivity, Leiden - Nijmegen, John Bendjamins Publishers, p. 23-48.

Kulikov L., 2009 , Evolution of case systems, in A. Malchukov \& A. Spenser (eds.), The Oxford handbook of case, Oxford University Press, p. 439-457.

Nikitina T., 2011, Pra-mande i niger-kongo : predvaritel'nye zamechanija k rekonstrukcii sintaksisa [Le Proto-Mandé et le Niger-Congo: Notes préliminaires sur la reconstruction du syntaxe], in E. V. Perekhval'skaya \& A. Ju. Zheltov (eds.), Le monde mandé. K 50-letiju V. F. Vydrina. Materialy ekspedicii v Zapadnuju Afriku (2001-...), Saint-Petersbourg, Nestor-Istorija, p. 33-39.

Paperno D., 2011, Grammaticheskij ocherk jazyka ben [Esquisse d'une grammaire de la langue beng], Acta Linguistica Petropolitana 7:2, p. 14-117.

Plungian V., 2000, Obshchaya morfologiya: Vvedenie $v$ problematiku [Morphologie générale : Une introduction], Moscou, Editorial URSS.

Vydrine V., 2007, Les adjectifs en dan-gwèètaa, Mandenkan 43, p. 77-103.

Vydrin V., 2009, Preverby v jazyke dan-gueta [Les préverbes en dan-gwè̀taa], Voprosy jazykoznanija 2, p. 75-84.

Vydrin V., 2010, "Nejtral'nyj vid" v dan-gueta i akcional'nyje klassy. [L'" aspect neutre » en dan-gwèètaa et l'Aktionsart], Voprosy jazykoznanija 5, p. 63-77.

Vydrine V. \& Mongnan A.K., 2008, Dictionnaire Dan-Français (dan de l'Est) avec une esquisse d'une grammaire du dan de l'Est et un index français-dan, Saint-Pétersbourg, Nestor-Istoria. 
APPENDICE

\begin{tabular}{|c|c|c|c|c|c|c|}
\hline Substantifs & Commun & Locatif & Inessif & Subessif & Adessif & Comitatif \\
\hline & \multicolumn{6}{|c|}{ NOMS LOCATIFS RÉGULIERS ET QUASI-RÉGULIERS } \\
\hline bléé & $\begin{array}{l}\text { bléédề } \\
\text { 'bord (du } \\
\text { champ)' }\end{array}$ & 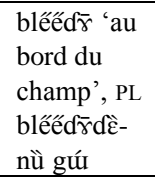 & & & & \\
\hline $6 \overline{0}$ & 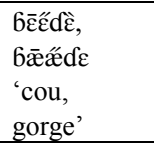 & 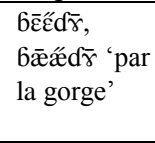 & $\begin{array}{l}6 \bar{\varepsilon} \ddot{\varepsilon}, 6 \bar{x} \not ́ \\
\text { 'par la } \\
\text { gorge, dans } \\
\text { la gorge' }\end{array}$ & $\begin{array}{l}\text { 6̄̄̄̀̀ 'sur } \\
\text { le cou' }\end{array}$ & & \\
\hline $\mathrm{d} \bar{\varepsilon} \ddot{\varepsilon}$ & $\begin{array}{l}\text { d } \bar{\varepsilon} \ddot{\varepsilon} \mathrm{d} \varepsilon \bar{\varepsilon} \\
\text { 'ruche' }\end{array}$ & $\begin{array}{l}\text { d } \bar{\varepsilon} \bar{\varepsilon} d \bar{\gamma} \\
\text { 'dans la } \\
\text { ruche' }\end{array}$ & & & & \\
\hline 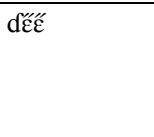 & 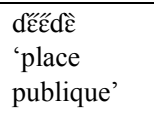 & 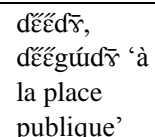 & & & & \\
\hline- & 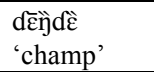 & $\begin{array}{l}\text { dē̄nd } \bar{y} \text { 'au } \\
\text { champ' }\end{array}$ & & & & \\
\hline 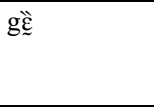 & $\begin{array}{l}\text { gę̄ỳd } \bar{\varepsilon} \\
\text { 'jambes, } \\
\text { pieds' }\end{array}$ & $\begin{array}{l}\text { gē̄̄d } \bar{\gamma} \text { 'aux } \\
\text { jambes, aux } \\
\text { pieds' }\end{array}$ & & 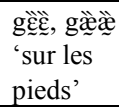 & & $\begin{array}{l}\text { g疋宦 } \\
\text { 'par/avec le } \\
\text { pied' }\end{array}$ \\
\hline gò & $\begin{array}{l}\text { gহ̣̄̂̀d } \\
\text { 'paillote' }\end{array}$ & $\begin{array}{l}\text { gā̄y } d \bar{\gamma} \\
\text { 'dans la } \\
\text { paillote' }\end{array}$ & & & & \\
\hline $\begin{array}{l}\text { gự̂́y 'endroit } \\
\text { sacré' }\end{array}$ & $\begin{array}{l}\text { gự̂́y̆ď 'case } \\
\text { sacrée' }\end{array}$ & $\begin{array}{l}\text { gự̂́y } \bar{\gamma} \\
\text { 'dans la } \\
\text { case sacrée' }\end{array}$ & & & & \\
\hline gblü & 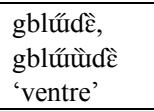 & $\begin{array}{l}\text { gblúfd'̄, } \\
\text { 'dans le } \\
\text { ventre' }\end{array}$ & & $\begin{array}{l}\text { gblǘú } \\
\text { 'dans le } \\
\text { ventre' }\end{array}$ & & \\
\hline (gwàà) & $\begin{array}{l}\text { gwààdề } \\
\text { 'rocher } \\
\text { plat' }\end{array}$ & $\begin{array}{l}\text { gwààd } \bar{\gamma} \text { 'au } \\
\text { rocher plat' }\end{array}$ & & & & \\
\hline- & $\begin{array}{l}\text { gwì̀jdê } \\
\text { 'vertex' }\end{array}$ & $\begin{array}{l}\text { gwì̀̀) } d \bar{\gamma}, \\
\text { gwìj dy } \\
\text { 'sur la tête, } \\
\text { sur le } \\
\text { sommet' }\end{array}$ & & $\begin{array}{l}\text { gwì̀y, } \\
\text { gbì̀j 'sur } \\
\text { la tête, } \\
\text { sur le } \\
\text { sommet' }\end{array}$ & & \\
\hline kèè & $\begin{array}{l}\text { kèèdê } \\
\text { 'occiput' }\end{array}$ & $\begin{array}{l}\text { kè̀dy' 'sur } \\
\text { l'occiput' }\end{array}$ & & & & \\
\hline
\end{tabular}




\begin{tabular}{|c|c|c|c|c|c|c|}
\hline Substantifs & Commun & Locatif & Inessif & Subessif & Adessif & Comitatif \\
\hline- & $\begin{array}{l}\text { kóíd̋̂̀ } \\
\text { 'hameau' }\end{array}$ & $\begin{array}{l}\text { kóíd̄' 'au } \\
\text { hameau' }\end{array}$ & & & & \\
\hline ḱ́ & $\begin{array}{l}\text { kóódê, } \\
\text { kógúudè } \\
\text { ‘maison', }\end{array}$ & $\begin{array}{l}\text { kóód̄' 'à la } \\
\text { maison', } \\
\text { 'dans la } \\
\text { maison' }\end{array}$ & & $\begin{array}{l}\text { kóò 'sur } \\
\text { le mur de } \\
\text { la } \\
\text { maison' }\end{array}$ & & \\
\hline kp๕̌̃ & 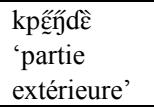 & 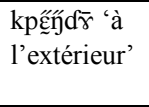 & 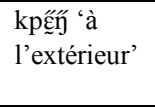 & & & \\
\hline 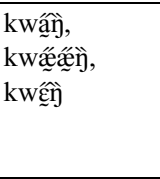 & 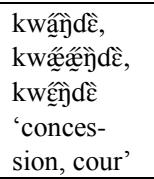 & 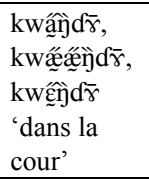 & & & & \\
\hline kwēế 'bras' & $\begin{array}{l}\text { kwē̌́cdè } \\
\text { 'poignet' }\end{array}$ & $\begin{array}{l}\text { kwē̌́d } \bar{\gamma} \\
\text { 'par le } \\
\text { poignet' }\end{array}$ & & & & \\
\hline- & 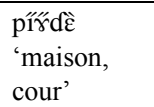 & $\begin{array}{l}\text { pí' } \text { '́d }^{\prime} \text { 'à la } \\
\text { maison, } \\
\text { chez X' }\end{array}$ & & & & \\
\hline $\mathrm{p} \hat{\gamma}$ & $\begin{array}{l}\text { pर̂rdè } \\
\text { 'village' }\end{array}$ & $\begin{array}{l}\text { p } \hat{\gamma} \mathrm{d} \bar{\gamma}, \text { pl'́r } \\
\text { 'au village' }\end{array}$ & $\begin{array}{l}\text { péé 'à ce } \\
\text { village' }\end{array}$ & & & \\
\hline 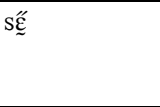 & sễ̂̀̀̀̃ 'côté' & 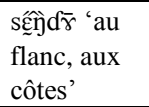 & & & & \\
\hline- & tààdề 'dos' & $\begin{array}{l}\text { tààd'̄, tàà } \\
\text { 'sur le dos, } \\
\text { au dos' }\end{array}$ & & & & \\
\hline- & 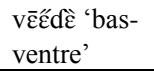 & $\begin{array}{l}\text { vē̌́d } \bar{\gamma} \bar{\gamma} \text { 'au } \\
\text { bas-ventre' }\end{array}$ & & & & \\
\hline $\mathrm{w} \bar{\Lambda}, \mathrm{w} \overline{\mathrm{D}}$ & $\begin{array}{l}\text { wh̄íd } \bar{\varepsilon} \\
\text { 'visage' }\end{array}$ & $\begin{array}{l}\text { wñ } \bar{\Lambda} \mathrm{d} \bar{\gamma} \text { 'au } \\
\text { visage' }\end{array}$ & & & & \\
\hline (yã्a) & yậa ‘œil’ & & $\begin{array}{l}\text { y }{ }_{\text {Nổ 'dans }} \\
\text { les yeux’ }\end{array}$ & $\begin{array}{l}\text { yậa ‘sur } \\
\text { l'œil' }\end{array}$ & & $\begin{array}{l}\text { yå̃a 'devant } \\
\text { les yeux' }\end{array}$ \\
\hline (yẫì) & yর̃̃ì̀ ‘soleil' & & & $\begin{array}{l}\text { y'̂́č, yáxæ̀ } \\
\text { 'au } \\
\text { soleil' }\end{array}$ & & \\
\hline- & 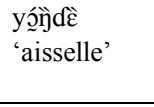 & 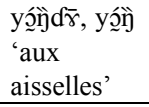 & & & & \\
\hline $\begin{array}{l}\text { zū 'bas, } \\
\text { fond ; fesses' }\end{array}$ & $\begin{array}{l}\text { zūuúudè } \\
\text { 'fesses' }\end{array}$ & $\begin{array}{l}\text { zūuúdō' ‘à } \\
\text { l'anus' }\end{array}$ & & & & \\
\hline & \multicolumn{6}{|c|}{ NOMS LOCATIFS INVARIABLES } \\
\hline
\end{tabular}




\begin{tabular}{|c|c|c|c|c|c|c|}
\hline Substantifs & Commun & Locatif & Inessif & Subessif & Adessif & Comitatif \\
\hline & $\begin{array}{l}\text { b’' } \\
\text { 'initiation' }\end{array}$ & $\begin{array}{l}\text { bə̋ 'à } \\
\text { l'initiation' }\end{array}$ & & & & \\
\hline & $\begin{array}{l}\text { búl, blû́ } \\
\text { 'brousse' }\end{array}$ & $\begin{array}{l}\text { bứ, blû́ } \\
\text { 'en } \\
\text { brousse' }\end{array}$ & & & & \\
\hline & dí 'bouche' & $\begin{array}{l}\text { dí' 'dans la } \\
\text { bouche' }\end{array}$ & & & & \\
\hline & \multicolumn{6}{|c|}{ NOMS LOCATIFS MIXTES } \\
\hline & $\begin{array}{l}\text { 6láàdđ̀̀ } \\
\text { 'champ', }\end{array}$ & $\begin{array}{l}\text { 6láá ‘au } \\
\text { champ' }\end{array}$ & & & & \\
\hline & 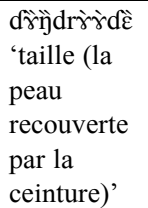 & $\begin{array}{l}\text { ḑ̌̀̀̀drò̀̀ } \\
\text { 'sous la } \\
\text { ceinture' }\end{array}$ & & & & \\
\hline & $\begin{array}{l}\text { d"óńkwn̂́̄odè } \\
\text { 'marché' }\end{array}$ & & & $\begin{array}{l}\text { d"’ókwwî́n } \\
\text { 'au } \\
\text { marché' }\end{array}$ & & \\
\hline & tô 'oreille' & $\begin{array}{l}\text { tőőd' ‘à } \\
\text { l'oreille' }\end{array}$ & & & & \\
\hline & yū 'nez’ & $\begin{array}{l}\text { yūụ́dȳ } \\
\text { 'dans le } \\
\text { nez' }\end{array}$ & & & & \\
\hline & \multicolumn{6}{|c|}{ NOMS LOCATIFS ASUFFIXAUX } \\
\hline gò 'tête' & $\begin{array}{l}\text { gòödè } \\
\text { 'emplacem } \\
\text { ent de la } \\
\text { source' }\end{array}$ & $\begin{array}{l}\text { gōōd } \bar{\gamma} \text { 'à la } \\
\text { source (du } \\
\text { fleuve)' }\end{array}$ & & $\begin{array}{l}\text { gàj̀, gì̀̀ } \\
\text { 'à la tête, } \\
\text { au } \\
\text { sommet' }\end{array}$ & & \\
\hline & $\begin{array}{l}\text { gbà } \\
\text { 'plafond, } \\
\text { grenier' }\end{array}$ & $\begin{array}{l}\text { gbà̀àd } d \bar{\gamma} \text { 'au } \\
\text { grenier' }\end{array}$ & & & & \\
\hline & $\begin{array}{l}\text { gwāa } \\
\text { 'mariage } \\
\text { (du point } \\
\text { de vue de la } \\
\text { femme)' }\end{array}$ & & & $\begin{array}{l}\text { gwāàa } \\
\text { gwāa bà : } \\
\text { dó gwāà } \\
\text { 'se } \\
\text { marier' }\end{array}$ & & \\
\hline & $\begin{array}{l}\text { kó ‘l'un } \\
\text { l'autre' }\end{array}$ & & $\begin{array}{l}\text { kóó 'l'un } \\
\text { dans } \\
\text { l'autre' }\end{array}$ & $\begin{array}{l}\text { kwî́i } \\
\text { 'ensem- } \\
\text { ble' }\end{array}$ & & $\begin{array}{l}\text { kw'̌́n } \\
\text { 'ensemble' }\end{array}$ \\
\hline & kò 'main' & & & kwė̀̀ỳ, & kòว̀ ‘sur & k̄̄̄ 'avec la \\
\hline
\end{tabular}




\begin{tabular}{|c|c|c|c|c|c|c|}
\hline Substantifs & Commun & Locatif & Inessif & Subessif & Adessif & Comitatif \\
\hline & & & & $\begin{array}{l}\text { kwè̀े } \\
\text { 'dans les } \\
\text { mains' }\end{array}$ & $\begin{array}{l}\text { les } \\
\text { mains' }\end{array}$ & main' \\
\hline & $\begin{array}{l}\text { kwè 'an, } \\
\text { année' }\end{array}$ & & & & & $\begin{array}{l}\text { kwē } \bar{\varepsilon} \text { 'cette } \\
\text { année' }\end{array}$ \\
\hline & sế 'terre' & 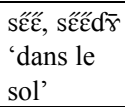 & $\begin{array}{l}\text { s'̌́ 'dans le } \\
\text { sol' }\end{array}$ & $\begin{array}{l}\text { síāā 'sur } \\
\text { le sol' }\end{array}$ & & \\
\hline & síx' 'feu' & & $\begin{array}{l}\text { sí's' 'dans } \\
\text { le feu, sur } \\
\text { le feu' }\end{array}$ & & & \\
\hline & s’ֵ ‘dent' & & & & & $\begin{array}{l}\text { sટ̃ 'avec 'avec } \\
\text { les dents' } \\
\text { (mordre) }\end{array}$ \\
\hline & $\begin{array}{l}\text { zị̄āāa } \\
\text { "chemin' }\end{array}$ & & & & 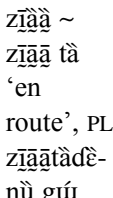 & \\
\hline
\end{tabular}

